\title{
Competing Inversion-Based Lasing and Raman Lasing in Doped Silicon
}

\author{
S. G. Pavlov, ${ }^{1,}$ N. Deßmann, ${ }^{2}$ B. Redlich, ${ }^{3}$ A. F. G. van der Meer, ${ }^{3}$ N. V. Abrosimov, ${ }^{4}$ H. Riemann, ${ }^{4}$ \\ R. Kh. Zhukavin, ${ }^{5}$ V. N. Shastin, ${ }^{5,6}$ and H.-W. Hübers ${ }^{1,2}$ \\ ${ }^{1}$ Institute of Optical Sensor Systems, German Aerospace Center (DLR), Berlin 12489, Germany \\ ${ }^{2}$ Department of Physics, Humboldt-Universität zu Berlin, Berlin 12489, Germany \\ ${ }^{3}$ Radboud University Nijmegen, Institute for Molecules and Materials, FELIX Laboratory, \\ Nijmegen 6525 ED, Netherlands \\ ${ }^{4}$ Leibniz-Institut für Kristallzüchtung (IKZ), 12489 Berlin, Germany \\ ${ }^{5}$ Institute for Physics of Microstructures, Russian Academy of Sciences, Nizhny Novgorod 603950, Russia \\ ${ }^{6}$ Lobachevsky State University, Nizhny Novgorod 603950, Russia
}

(Received 8 January 2018; revised manuscript received 20 July 2018; published 5 October 2018)

\begin{abstract}
We report on an optically pumped laser where photons are simultaneously generated by population inversion and by stimulated Raman scattering in the same active medium, namely crystalline silicon doped by bismuth ( $\mathrm{Si}: \mathrm{Bi})$. The medium utilizes three electronic levels: ground state $\left[|1\rangle: 1 s\left(A_{1}\right)\right.$ in $\left.\mathrm{Si}: \mathrm{Bi}\right]$, upper $\left[|3\rangle: 2 p_{ \pm}\right]$and lower $[|2\rangle: 1 s(E)]$ laser levels. The $|1\rangle \leftrightarrow|3\rangle$ and $|2\rangle \leftrightarrow|3\rangle$ transitions are optically allowed and the $|1\rangle \leftrightarrow|2\rangle$ transition is Raman active. Lasing based on population inversion occurs between the states $|3\rangle$ and $|2\rangle$, while Raman scattering benefits from the Raman-active transition. At high pump power the inversion-based stimulated emission $|3\rangle \rightarrow|2\rangle$ disappears, because electronic scattering from $|1\rangle$ to $|2\rangle$ via a virtual state dominates and the electrons are excited into $|2\rangle$ rather than into $|3\rangle$. Starting as population inversion-based lasing, it ends as stimulated Raman scattering. Our model shows that such a competition occurs on the timescale of the 10-ps-long pump pulse.
\end{abstract}

DOI: $10.1103 /$ PhysRevX.8.041003

Subject Areas: Condensed Matter Physics,

Nonlinear Dynamics, Photonics

Optically pumped lasers are examples of active media where two alternative lasing schemes can be realized: inversion based and inversionless. Population inversion lasing (PIL) is based on inversion between particular states, an upper long-lived electronic state $|3\rangle$ and a lower shortlived electronic state $|2\rangle$. The transition between these states is usually optically allowed. Two lasing mechanisms without population inversion are known. These are light amplification by quantum interference between electronic states [1] or by stimulated Raman scattering (SRS) [2-4]. When an optically allowed PIL transition, $|3\rangle \rightarrow|2\rangle$, and a SRS transition, $|1\rangle \rightarrow|2\rangle$ via a virtual state, are combined in a three-level cascade $|3\rangle \rightarrow|2\rangle \rightarrow|1\rangle$, alternative lasing schemes can occur.

Such schemes have been first considered in optically pumped, low-dimensional semiconductor structures with intersubband $|3\rangle \rightarrow|2\rangle \rightarrow|1\rangle$ cascades. Theoretical models of the total gain in a three-level system, including

\footnotetext{
*sergeij.pavlov@dlr.de
}

Published by the American Physical Society under the terms of the Creative Commons Attribution 4.0 International license. Further distribution of this work must maintain attribution to the author(s) and the published article's title, journal citation, and DOI. methods based on the density matrix theory, show that at certain conditions, such as fast resonant scattering and large pump powers, the Raman gain can exceed the gain on transitions between the same levels with population inversion [5-7]. Both PIL and SRS lasing mechanisms have been experimentally achieved in different active media having three electronic subbands as laser levels [8-10]. PIL and SRS lasing mechanisms can be distinguished since the emission frequency of the Raman laser changes linearly with the pump laser frequency while it is independent of the pump laser frequency for PIL. Raman lasing at infrared wavelengths has been obtained from artificial three-level quantum-well structures, e.g., in $\mathrm{GaAs} /(\mathrm{Al}, \mathrm{Ga}) \mathrm{As}$ and $(\mathrm{Ga}, \mathrm{In}) \mathrm{As} /(\mathrm{Al}, \mathrm{In}) \mathrm{As}[8,10]$. SRS lasing occurs on the $|1\rangle \rightarrow|2\rangle$ transition by optical excitation on the $|1\rangle \rightarrow|3\rangle$ transition while the spacing between states $|1\rangle$ and $|2\rangle$ corresponds to a phonon energy or an electronic resonance. Similar structures were used for quantum fountain lasers [9]. In these lasers PIL on the $|3\rangle \rightarrow|2\rangle$ transition dominates. An electrically excited infrared Raman laser has been claimed by embedding a three-level structure in the active medium of an infrared quantum-cascade laser which serves as the optical pump [3]. It should be noted that simultaneous inversion and Raman lasing has not been obtained in these devices with electronic subbands. However, for an accurate description electron scattering with a broad kinetic 
energy spectrum and the formation of hot electrons in the subbands should be taken into account [8]. Separate PIL and Raman lasing have been demonstrated using atomiclike discrete levels of shallow donor centers in silicon [11,12].

In a three-level active medium, strong competition between PIL and SRS lasing mechanisms can occur since the lasing gains of both mechanisms are dominantly determined by population differences between the states in the cascade $|3\rangle \rightarrow|2\rangle \rightarrow|1\rangle$, namely, $n_{3}$ vs $n_{2}$ for PIL and $n_{1}$ vs $n_{2}$ for SRS. As a result, saturation occurs at different optical pump powers and the pump intensity is the parameter which controls the relative contributions of PIL and SRS to the stimulated emission. In this paper, we show that such a three-level system is formed by the electronic states of the bismuth donor in silicon ( $\mathrm{Si}: \mathrm{Bi})$. This active medium supports the simultaneous operation of terahertz (THz) PIL and SRS lasing under specific optical pump conditions with the infrared radiation from a free-electron laser (FEL). Low pump rates and resonant pumping into the upper level favor the PIL mechanism while the SRS lasing benefits from high optical pump intensities and slightly detuned off-resonant pumping (Fig. 1). The high- $Q$ factor of the $\mathrm{Si}: \mathrm{Bi}$ laser resonator supports lasing photons between pump micropulses and enables thus the observation of the competing laser processes despite the short (approximately $10 \mathrm{ps)} \mathrm{pump} \mathrm{pulses} \mathrm{and} \mathrm{the} \mathrm{large} \mathrm{pulse}$ separation (1 ns).

As an active medium, several $n$-type silicon crystals grown by the float-zone technique in the $\{100\}$ direction have been used. The dopant concentration in the crystals is in the range of $N_{D} \sim(3-5) \times 10^{15} \mathrm{~cm}^{-3}$. Samples with dimensions of $(7 \times 7) \times 5 \mathrm{~mm}^{3}$ were cut from the ingot and optically polished to form a high- $Q$ resonator operating on total internal reflection modes. The $\mathrm{Si}$ : Bi samples were first characterized by measuring their impurity absorption spectra at low temperature (about $5 \mathrm{~K}$ ) with a Fourier transform infrared spectrometer (Bruker Vertex 80v) and a spectral resolution of $0.1 \mathrm{~cm}^{-1}(12.5 \mu \mathrm{eV})$. The lasing experiments were carried out with the infrared free-electron laser FELIX at Radboud University, Nijmegen, The Netherlands. Its pump pulses consist of $6-\mu$ s-long macropulses at a repetition rate of $5 \mathrm{~Hz}$. Each macropulse consists of approximately 10 -ps-long micropulses with a peak power of about $10 \mathrm{MW}$ separated by $1 \mathrm{~ns}$. The spectral width of each micropulse is approximately $0.5 \mathrm{meV}$ (full width at half maximum, FWHM). The Si samples were mounted in a cryogenic dipstick, which was immersed in a liquid-helium transport vessel. The dependence of the spectrally integrated $\mathrm{Si}$ : Bi laser intensity on the FEL pump photon energy has been measured in the entire impurity absorption band up to the transition into the bottom of conduction band [spectral resolution of $0.05 \mu \mathrm{m}$ (FWHM) as determined by a grating spectrometer in the FEL diagnostic station]. The lasing emission from $\mathrm{Si}$ was

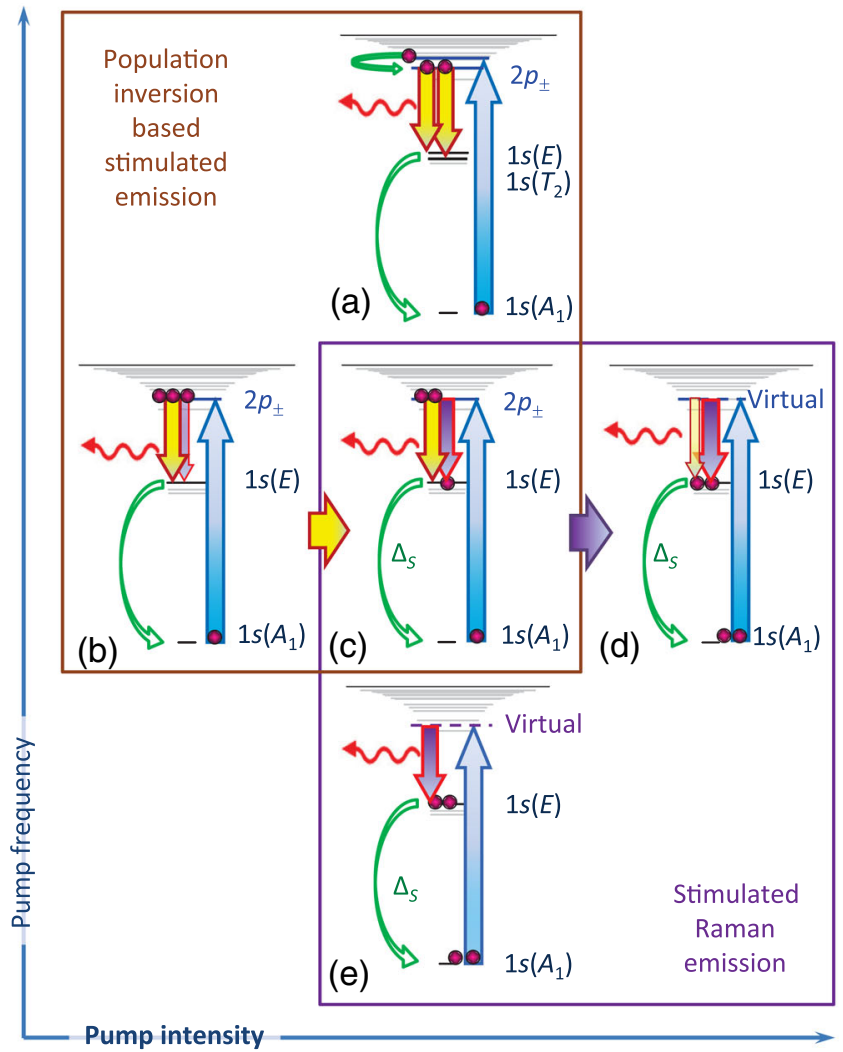

FIG. 1. Different lasing schemes in Si:Bi lasers. The transition between these schemes depends on the pump frequency, pump intensity, and time that has elapsed after the start of the pump pulse. The horizontal gray and black lines indicate the discrete energy levels of the Bi donor. The straight blue arrows up indicate the optical pump process. The straight arrows down are for PIL (filled yellow) and SRS (filled purple) lasing, the curved arrows down are the dominating nonradiative relaxation paths of an excited electron. $1 s(E)$ and $1 s\left(T_{2}\right)$ are split-off ground states, $1 s(E) \rightarrow 1 s\left(A_{1}\right)$ is a Raman-active intracenter transition in $n$-Si with an energy $\Delta_{S}$. (a) PIL: four-level laser scheme based on population inversion between the long-lived $2 p_{ \pm}$and the shortlived $1 s(E), 1 s\left(T_{2}\right)$ states; depletion of the lower laser states into the ground state is due to intervalley phonons. (b)-(d) merged PIL and SRS lasing processes under resonant pumping into the longlived $2 p_{ \pm}$state. In this particular case the virtual state coincides with the $2 p_{ \pm}$state of the $\mathrm{Bi}$ donor. At pump start (b) PIL dominates, the laser scheme is reduced to the three levels; later on (c) SRS develops and balances PIL; in the end (d) SRS prevails, and the elecrons are scattered directly into the $1 s(E)$ state, the upper $2 p_{ \pm}$level becomes essentially unpopulated. (e) SRS lasing occurs at off-resonant pumping to any of the higher impurity states.

analyzed by a Fourier transform infrared spectrometer operating in step-scan mode with a spectral resolution of $0.3 \mathrm{~cm}^{-1}(37 \mu \mathrm{eV})$ equipped with a $\mathrm{Ge}:$ Ga photoconductive detector, which is sensitive from 40 to $120 \mu \mathrm{m}$. The interferograms were measured with a time resolution of $50 \mathrm{~ns}$. This time gating has reduced significantly the integrated laser intensity reaching the detector, so that only 


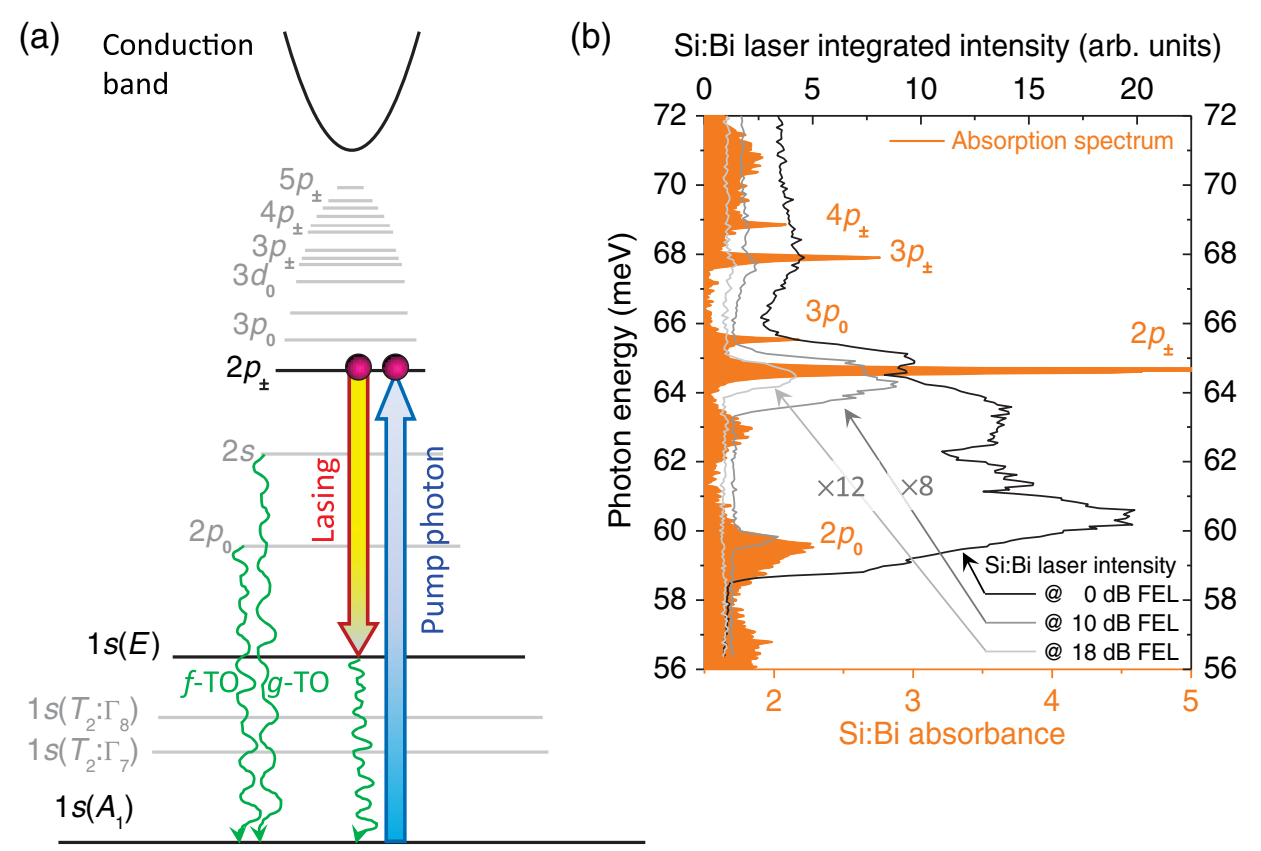

FIG. 2. (a) Si:Bi laser scheme under resonant pumping in the upper laser level. Labels are the ground and excited states of a Bi center. Bold straight arrows indicate optical pump and lasing transitions, wavy arrows show nonradiative phonon-assisted relaxations. Note that energy spacings below the $2 p_{0}$ state are for clarity not to scale. (b) Integrated intensity of the $\mathrm{Si}$ : $\mathrm{Bi}$ laser as a function of the pump photon energy (spectral resolution: $0.05 \mu \mathrm{m}$ ). The laser spectra were measured at different FEL power (the attenuation from maximum FEL power is given in the legend). For comparison the $\mathrm{Si}$ : Bi absorption spectrum measured with a spectral resolution of $0.1 \mathrm{~cm}^{-1}$ $(12.5 \mu \mathrm{eV})$ is plotted as well. Note that at lower pump intensities the integrated intensity is resolved into a discrete spectrum which is similar to the impurity absorption spectrum.

stimulated emission at pump powers well above the lasing threshold could be measured. Spectra were calculated from the interferograms applying a Blackman-Harris filter for apodization. Combinations of infrared filters mounted in the dipstick at liquid-helium temperature and outside at room temperature prevented that pump radiation from reaching the detector.

The hydrogenlike $\mathrm{Bi}$ centers in $\mathrm{Si}$ form localized electronic states in the band gap. The degenerate $1 \mathrm{~s}$ electronic ground state, whose orbit is closest to the nucleus, is affected by the so-called chemical splitting which leads to a splitting and shifting: the singlet $1 s\left(A_{1}\right)$ state (laser level $|1\rangle$ ) is most strongly downshifted from the position predicted by the effective mass theory while the triplet $1 s\left(T_{2}\right)$ and doublet $1 s(E)$ (laser level $|2\rangle$ ) components are less downshifted [Fig. 2(a)]. Optical transitions between these states are dipole forbidden while the transition between the $1 s\left(A_{1}\right)$ ground state and the excited $1 s(E)$ state $|1\rangle \leftrightarrow|2\rangle$ is Raman active [13]. In thermal equilibrium at low temperature, typically less than $10 \mathrm{~K}$, virtually all extrinsic electrons are bound to the donor ground state.

An optically pumped intracenter $n$-Si:Bi laser [Figs. 1 and 2(a)] utilizes population inversion formed by the accumulation of electrons, photoexcited from the donor ground state $(|1\rangle \rightarrow|3\rangle)$ and accumulated in the long-lived
$2 p_{ \pm}$state (laser level $|3\rangle$, lifetime about 50 ps [14]). The $2 p_{0}$ and $2 s$ excited states in $\mathrm{Si}: \mathrm{Bi}$ are resonantly coupled to the ground state via intervalley $f$-TO and $g$-TO optical phonons [Fig. 2(a)]. These states remain unpopulated [15] and can therefore be neglected when considering the PIL scheme. Therefore, the $\mathrm{Si}: \mathrm{Bi}$ laser emits on the $2 p_{ \pm} \rightarrow$ $1 s(E)$ transition $(|3\rangle \rightarrow|2\rangle)$ with the corresponding photon energy $h \omega_{\mathrm{PIL}}=\left|E_{2 p \pm}-E_{1 s(E)}\right|(23.8 \mathrm{meV})$ and, when pumped above the upper laser state $[|1\rangle \rightarrow|4\rangle$, Figs. 1(a), 3(b), 4(a)], additionally on the $2 p_{ \pm} \rightarrow 1 s\left(T_{2}\right)$ transition with $h \omega_{2}=\left|E_{2 p \pm}-E_{1 s(T 2)}\right|(25.5 \mathrm{meV})$ [15]. This modification of the $\mathrm{Si}: \mathrm{Bi}$ lasing spectrum occurs due to specific coherent excitation of the upper laser state. Under resonant pumping in the $2 p_{ \pm}$state (which has the irreducible representation $2 T_{1}+2 T_{2}$ of $T_{d}$ symmetry group) only two asymmetric from four components of the $2 p_{ \pm}$state are selectively excited that results in dominating dipole-allowed laser emission on $2 p_{ \pm} \rightarrow 1 s(E)$ transition. When pumped above the $2 p_{ \pm}$state, this pumping induced "selectivity" gets lost due to electron scattering during relaxation towards the $2 p_{ \pm}$state (also including processes between equivalent conduction band valleys). Therefore, all components of the $2 p_{ \pm}$Bi state become equally populated and both dipole-allowed $2 p_{ \pm}\left(2 T_{1}+2 T_{2}\right) \rightarrow$ $1 s(E) ; 1 s\left(T_{2}\right)$ laser transitions occur. Depletion of the lower laser state is dominated by an interaction with intervalley 

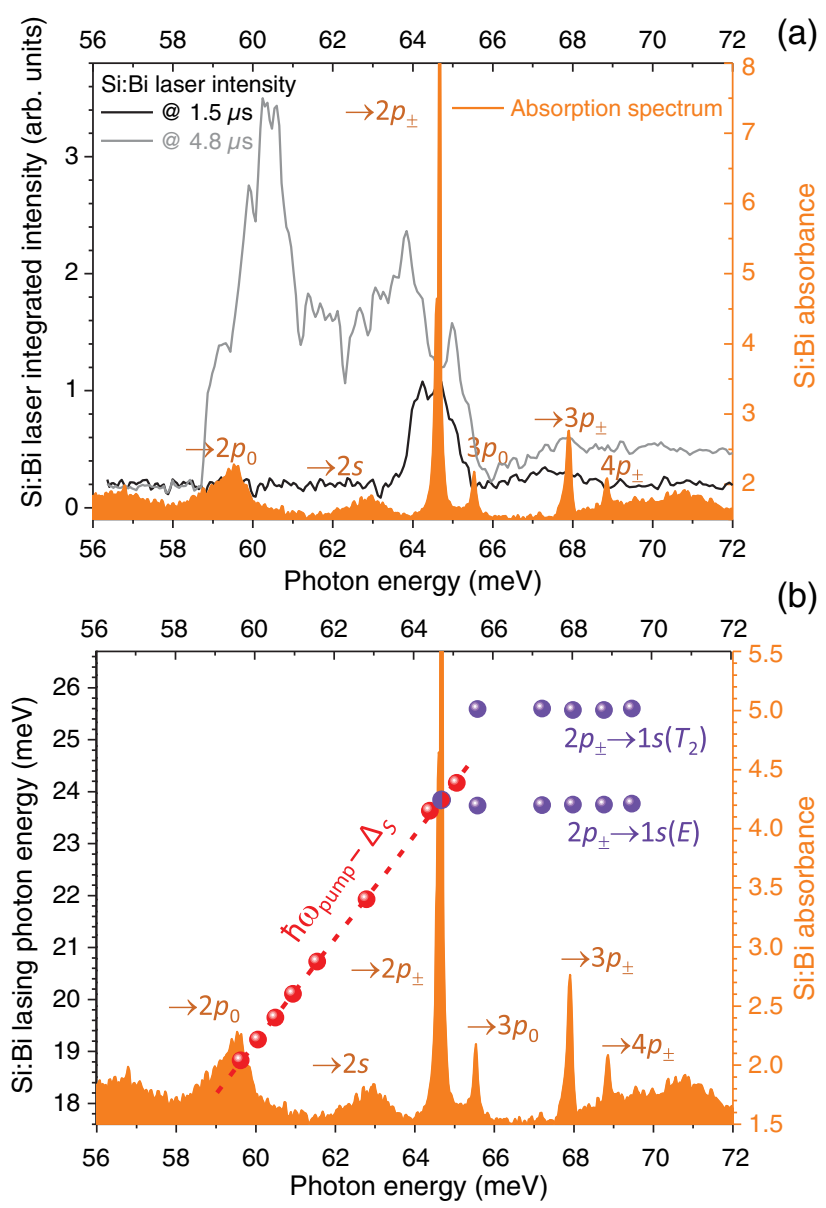

FIG. 3. (a) Integrated intensity of the Si:Bi laser vs FEL pump photon energy at different times elapsed from the front of the pump pulse and compared with the $\mathrm{Si}: \mathrm{Bi}$ absorption spectrum. Note that the $\mathrm{Si}: \mathrm{Bi}$ lasing starts on the $1 s\left(A_{1}\right) \rightarrow 2 p_{ \pm}$transition where pumping is most effective for the PIL transition $1 s\left(A_{1}\right) \rightarrow$ $2 p_{ \pm}$(at about $64.6 \mathrm{meV}, 19.2 \mu \mathrm{m}$ ). With a delay of a few microseconds the SRS-typical, broad lasing spectrum sets on and dominates towards the end of the pump macropulse (see also complementary file Si_Integrated_Emission.gif for full reconstructed dynamics [18]). The spectral resolution of pump spectra is $0.05 \mu \mathrm{m}$ and of absorption spectrum is $0.1 \mathrm{~cm}^{-1}$. (b) Dependence of the photon energy and intensity of the SRS and PIL emission on the pump photon energy. The $\mathrm{Si}$ :Bi absorption spectrum is given for orientation. The circles show the pump photon energies where $\mathrm{Si}$ : Bi emission spectar were taken. Note the linearity of the SRS lasing frequency with the pump photon energy and the Stokes shift of about $40.8 \mathrm{meV}$. The SRS lasing (dashed line) spans the pump photon range from below the $2 p_{0}$ excited state up to the $3 p_{0}$ excited state. Pumping in the $2 p_{ \pm} \mathrm{Bi}$ state results in joint PIL and SRS lasing. Pumping in the $3 p_{0} \mathrm{Bi}$ state and above results in PIL only.

phonons [16]. In general, a four-level laser scheme is realized [Figs. 1(a) and 4(a)]. This laser scheme involves the donor ground state $|1\rangle$, the pumped state $|4\rangle$, and the upper $|3\rangle$ and lower $|2\rangle$ laser states. A particular situation occurs when the pumped state and the upper laser state are identical $|4\rangle \equiv|3\rangle$
[Figs. 1(b),1(c),1(d), 4(b)]. This is a degenerate four-level scheme with three electronic states involved in the laser mechanism. The lower $1 s(E) \rightarrow 1 s\left(A_{1}\right)$ intracenter transition in the three-level system provides resonant inelastic light scattering and by this enhances SRS in $n$-Si, in particular in $\mathrm{Si}: \mathrm{Bi}$ [12]. Under nonresonant excitation a virtual state is formally ascribed to an optically excited electron. In Raman Stokes lasers the energy of the emitted photon $h \omega_{\text {SRS }}$ equals the pump photon energy $h \omega_{\text {pump }}$ reduced by the Stokes shift $\Delta_{S}: h \omega_{\mathrm{SRS}}=h \omega_{\text {pump }}-\Delta_{S}$. In infrared Raman lasers [2] the Stokes shift corresponds to the zone-centered optical phonon, whereas for THz Raman lasers it is the energy of the Raman active $1 s(E) \rightarrow 1 s\left(A_{1}\right)$ transition, $\Delta_{S}=\left|E_{1 s(A 1)}-E_{1 s(E)}\right|$ [17]. This is the outgoing resonance of the electronic Raman scattering [Figs. 1(c), 1(d), 1(e)], which has been observed for all group- $\mathrm{V}$ donors in $\mathrm{Si}$ as stimulated Raman emission under optical pumping by radiation from an infrared FEL [12,15,17]. Under resonant excitation $|1\rangle \rightarrow|3\rangle$, the virtual state coincides with an electronic state, $\mid$ virtual $\rangle \equiv|3\rangle$, and a so-called incoming resonance appears for SRS [Figs. 1(c), 1(d), 2(a)]. As a result, the emission frequencies of the two different mechanisms, SRS and PIL, are identical $\omega_{\text {SRS }} \equiv \omega_{\text {PIL }}$.

We have analyzed $\mathrm{Si}: \mathrm{Bi}$ emission by tuning the FEL pump frequency across the absorption spectrum of the $\mathrm{Bi}$ donors and measuring the integrated intensity of the $\mathrm{Si}: \mathrm{Bi}$ laser [Fig. 2(b)]. At the highest pump power there is almost continuous $\mathrm{Si}$ :Bi laser emission for FEL pump photon energy from 58 to $66 \mathrm{meV}$, as expected for SRS. When attenuating the pump power, laser emission remains observed only when pumped resonantly into the $2 p_{0}$ and $2 p_{ \pm}$states (at $10 \mathrm{~dB}$ FEL attenuation) and only in the $2 p_{ \pm}$ state (at $18 \mathrm{~dB}$ FEL attenuation) [Fig. 2(b)]. In Fig. 3(a) the integrated intensity of the $\mathrm{Si}: \mathrm{Bi}$ laser is shown when measured in the time gates set at 1.5 and $4.8 \mu$ s after the onset of the pump pulse. Early lasing appears only when pumped at around $64.6 \mathrm{meV}$, which corresponds to the $1 s\left(A_{1}\right) \rightarrow 2 p_{ \pm}$Bi transition. At $4.8 \mu \mathrm{s}$ laser emission occurs not only when pumped resonantly into a bound state but also between the states. This is an indication of SRS lasing. Figure 3(b) summarizes these findings. The $\mathrm{Si}$ : Bi laser photon energy increases linearly with the pump photon energy up to about $24.5 \mathrm{meV}$ at $65.5 \mathrm{meV}$ pump photon energy. Above that pump photon energy, emission occurs only when $\mathrm{Si}: \mathrm{Bi}$ is directly excited into one of the $\mathrm{Bi}$ states. The emission frequency corresponds either to the $2 p_{ \pm} \rightarrow 1 s\left(T_{2}\right)$ transition or to the $2 p_{ \pm} \rightarrow 1 s(E)$ transition. In this case PIL prevails.

The distinction between PIL and SRS becomes possible by analyzing the $\mathrm{Si}$ : Bi lasing thresholds, output power, and emission dynamics if compared with the borderline cases, i.e., resonant pumping in other electronic states around the $2 p_{ \pm}$state (pure PIL, pumping $|1\rangle \rightarrow|4\rangle$ ) or off-resonance pumping (SRS, pumping $|1\rangle \rightarrow \mid$ virtual state $\rangle)$. PIL has a lower lasing threshold and remains at strongly reduced FEL 
PIL
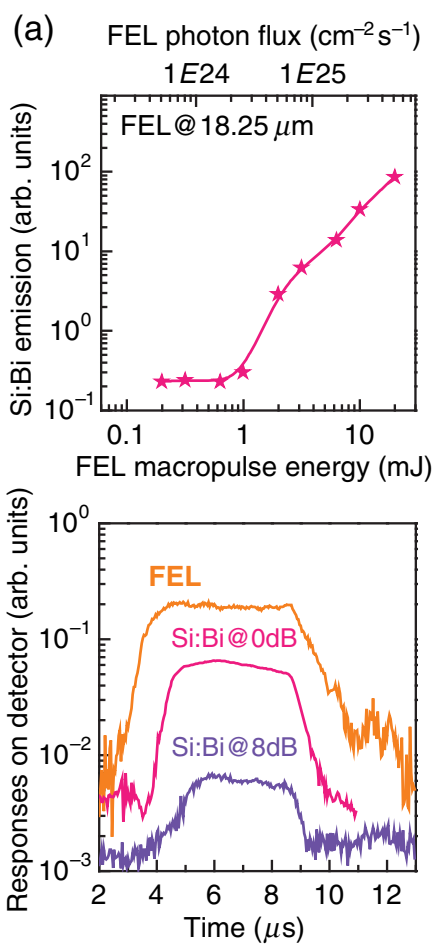

Emission frequency $(\mathrm{THz})$

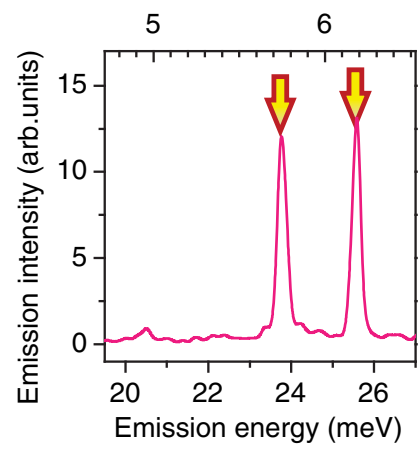

$P I L+S R S$

(b) FEL photon flux $\left(\mathrm{cm}^{-2} \mathrm{~s}^{-1}\right)$
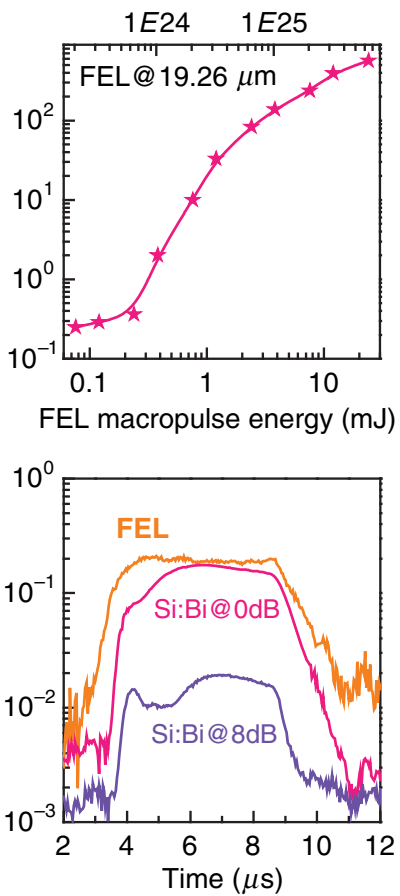

Emission frequency $(\mathrm{THz})$

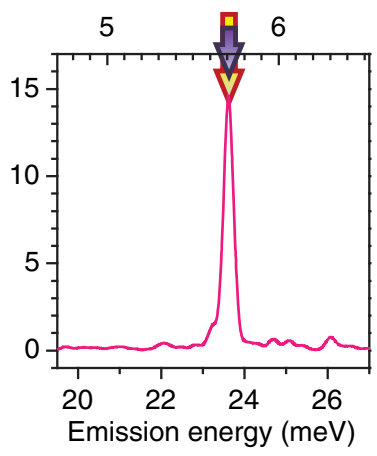

SRS

(c) FEL photon flux $\left(\mathrm{cm}^{-2} \mathrm{~s}^{-1}\right)$
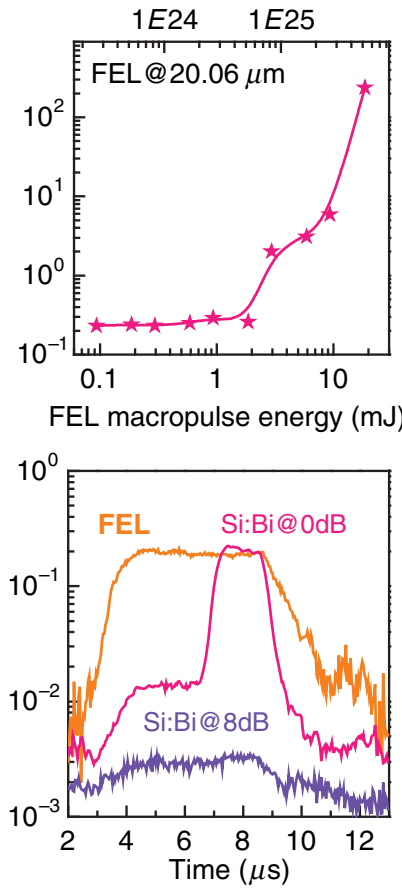

Emission frequency $(\mathrm{THz})$

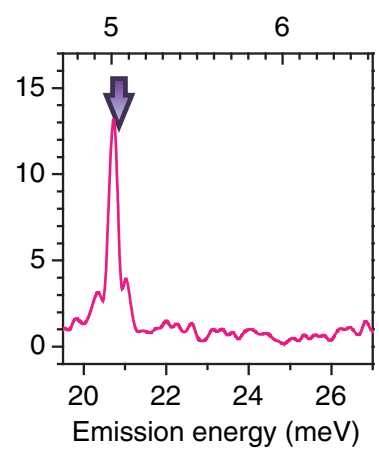

FIG. 4. Lasing thresholds, dynamics, and emission spectra for different laser mechanisms in Si:Bi. (a) Typical four-level population inversion scheme (PIL): The pump photon energy corresponds to the excitation of bound electrons into a higher excited state. The laser transitions are $2 p_{ \pm} \rightarrow 1 s(E)$ and $2 p_{ \pm} \rightarrow 1 s\left(T_{2}\right)$ at 5.75 and $6.16 \mathrm{THz}$, correspondingly. The FEL pump wavelength is $18.25 \mu \mathrm{m}$ (16.43 THz or $67.95 \mathrm{meV}$ ) and the photon flux is $4 \times 10^{25} \mathrm{~cm}^{-2} \mathrm{~s}^{-1}$. (b) Simultaneous operation of Raman and population inversion lasing (PIL and SRS): The pump photon energy corresponds to excitation in the $2 p_{ \pm} \mathrm{Bi}$ state. The previously distinct laser lines merge into one emission line which corresponds to the energy of the $2 p_{ \pm} \rightarrow 1 s(E)$ Bi transition. The FEL pump wavelength is $19.26 \mu \mathrm{m}$ (15.57 THz or $64.4 \mathrm{meV}$ ) and the photon flux is $4 \times 10^{25} \mathrm{~cm}^{-2} \mathrm{~s}^{-1}$. (c) Raman lasing scheme (SRS): The pump photon energy corresponds to excitation into a virtual state. The Stokes emission line is from the virtual state into the $1 s(E)$ state $(5.04 \mathrm{THz}$ or $20.84 \mathrm{meV})$. The FEL pump wavelength is $20.06 \mu \mathrm{m}(14.95 \mathrm{THz}$ or $61.81 \mathrm{meV})$ and the photon flux is $5 \times 10^{25} \mathrm{~cm}^{-2} \mathrm{~s}^{-1}$.

pump powers where SRS has ceased [Fig. 2(b)]. Pure PIL [Fig. 4(a)] differs from the merged case [Fig. 4(b)] by the number of emitted lines, namely two lines at $23.8 \mathrm{meV}$ $\left[2 p_{ \pm} \rightarrow 1 s(E)\right]$ and $25.5 \mathrm{meV}\left[2 p_{ \pm} \rightarrow 1 s\left(T_{2}\right)\right]$ while in the merged case only the line at $23.8 \mathrm{meV}$ exists. The SRS lasing [Fig. 4(c)] differs from PIL by having only one emission line (for instance, about $20.8 \mathrm{meV}$ at a pump wavelength $20.06 \mu \mathrm{m}$, photon energy of about $61.8 \mathrm{meV}$ ) which frequency does not correspond to any Bi intracenter transition. In addition, there is a significant delay of the $\mathrm{Si}$ : Bi lasing pulse relative to the pump pulse [for instance, about $3 \mu \mathrm{s}$ at a pump wavelength $20.06 \mu \mathrm{m}$ vs $1 \mu \mathrm{s}$ at a pump wavelength $18.25 \mu \mathrm{m}$, Fig. 4(a) and 4(c), middle row], the lasing threshold is larger, and the slope efficiency is different. Such a delay in the SRS pulse is often reflected in a steplike dynamic transition from spontaneous to stimulated mode, more pronounced at pump wavelengths with a larger SRS lasing threshold. Within the macropulse 

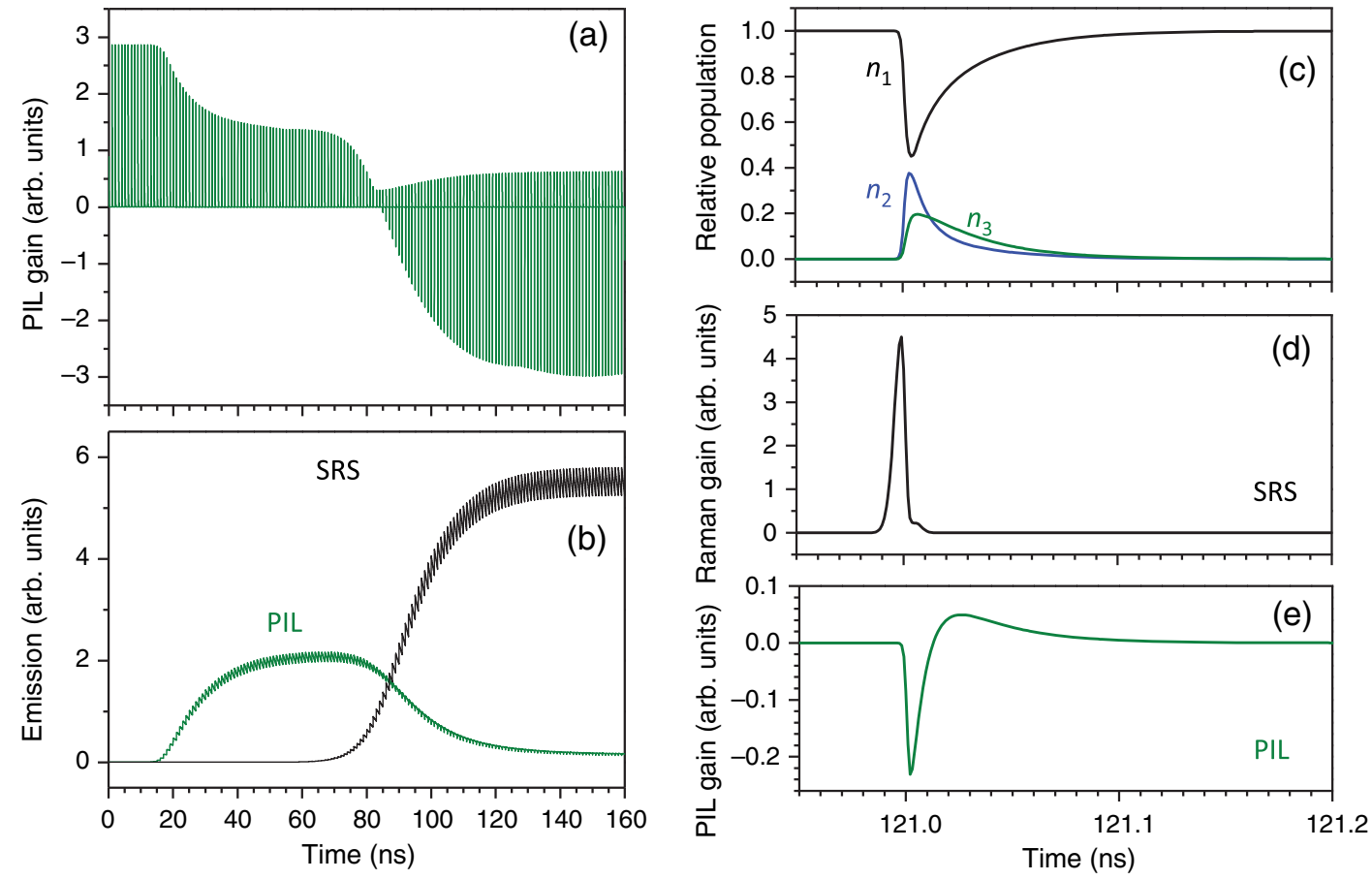

FIG. 5. Simulated laser pulse dynamics of a three-level system under pulsed FEL excitation at the front of the macropulse: (a) Evolution of the PIL gain; (b) separate outputs of the PIL and SRS lasing. Evolution on the micropulse timescale: (c) relative populations of the states, (d) SRS gain, (e) PIL gain. The parameters of the model: pump rate $R_{13}$ is $10^{11} \mathrm{~s}^{-1}$; the relaxation rate $W_{32}$ between the upper $2 p_{ \pm}$state and the intermediate $1 s(E)$ state is $2 \times 10^{10} \mathrm{~s}^{-1}$ and $W_{21}$ between the $1 s(E)$ and the $1 s\left(A_{1}\right)$ ground state is $10^{11} \mathrm{~s}^{-1}$; direct relaxation from the upper into the ground state $(|3\rangle \rightarrow|1\rangle)$ is neglected; losses at the Si:Bi emission frequency are $10^{10} \mathrm{~s}^{-1}$. Note that developing Raman emission competes with the PIL and causes a specific step in the output power (for details see the Supplemental Material [18]).

pump time, the merged case [Fig. 4(b)] combines features of both lasing mechanisms resulting in a single $2 p_{ \pm} \rightarrow$ $1 s(E)$ emission line, low lasing threshold and little delay of the emission pulse as for PIL. However, the emission pulse has a characteristic step in the first part, which becomes more pronounced at low FEL pump power, indicating changes of the laser dynamics.

We explain the observed lasing dynamics by evolution of the intracavity stimulated emission due to fast changes in level populations, which we have modeled on the basis of balanced equations for a three-level system (see Supplemental Material [18]). The PIL gain $g_{\mathrm{PIL}}$ is proportional to the pump-induced population difference $n_{3}-n_{2}$. It saturates when the absorption on the $1 s\left(A_{1}\right) \rightarrow 2 p_{ \pm}$ $(|1\rangle \rightarrow|3\rangle)$ transition saturates, i.e., when $n_{3}$ approaches $n_{1}$. The laser emission on the $2 p_{ \pm} \rightarrow 1 s(E)(|3\rangle \rightarrow|2\rangle)$ transition saturates when $n_{2}$ approaches $n_{3}$ in the PIL mode. The Raman gain is proportional to the population difference $n_{1}-n_{2}$ and to the pump power [5]. Saturation of the SRS emission occurs by stimulated electron scattering into the $1 s(E)$ state when $n_{2}$ approaches $n_{1}$. Under pulsed pumping the decay of the PIL gain is determined by the lifetime of level $|3\rangle$ (about 50 ps), while the SRS gain exists only during the pump micropulse. The $Q$ factor of the Si.Bi laser resonator plays a key role, because it enables us to sustain stimulated emission between the approximately 10-ps-long pump micropulses which have an interpulse period of $1 \mathrm{~ns}$. The typical photon lifetime in the $\mathrm{Si}$ resonator under intracenter pumping is about $10^{-8} \mathrm{~s}$. It is limited mainly by lattice absorption and radiative losses. Thus, averaging over a 1-ns period yields relatively lower Raman gain and causes the delayed onset of the SRS emission [Fig. 4(c)] as compared to PIL [Fig. 4(a)]. The model shows that at certain pump rates into the upper level $2 p_{ \pm}$and typical optical cross sections for transitions in $\mathrm{Si}$ :Bi, SRS can block the initial absorption of pump light from the lowest level $|1\rangle$ into level $|3\rangle$ and dominate over PIL (Fig. 5). The timescale of the process is similar to the pump micropulse duration. The upper long-lived level $|3\rangle$, populated in the PIL scheme by optical absorption, becomes a virtual state in the SRS process where electrons from the ground state $|1\rangle$ scatter directly in the lower laser state $|2\rangle$ leaving level $|3\rangle$ unpopulated. The growing gain for SRS emission leads to a decay of the PIL gain and emission. This causes the characteristic step in the $\mathrm{Si}: \mathrm{Bi}$ laser output power under resonant pumping [Fig. 5(b)].

A slight detuning of the pump wavelength from the resonance with the $1 s\left(A_{1}\right) \rightarrow 2 p_{ \pm}$transition allows distinguishing between the two lasing mechanisms by timeresolved emission spectroscopy. Figure 6 demonstrates the 


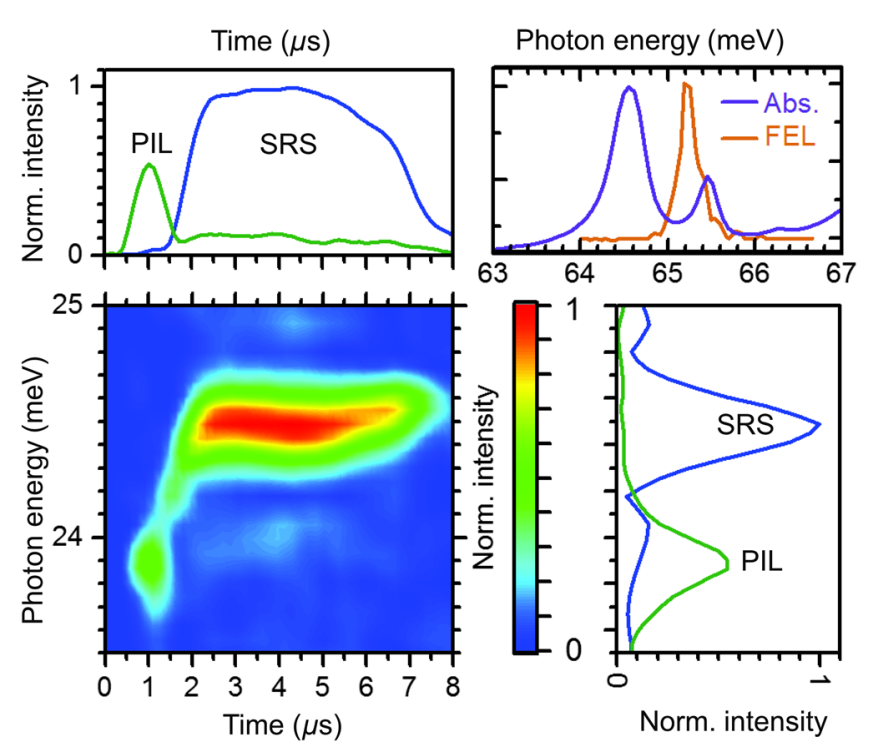

FIG. 6. (See also the complementary files with reconstructed dynamics [18].) Temporal and spectral evolution of the Si:Bi laser emission when pumped at $19.02 \mu \mathrm{m}(65.2 \mathrm{meV})$ which is $0.6 \mathrm{meV}$ above the long-lived $2 p_{ \pm}$state (color map with projections to time and photon energy axis). The emission lines at approximately $23.8 \mathrm{meV}(5.78 \mathrm{THz})$ and at $24.5 \mathrm{meV}$ $(5.92 \mathrm{THz})$ correspond to the PIL $2 p_{ \pm} \rightarrow 1 s(E)$ transition and to the Raman emission from a virtual state into the $1 s(E)$ state, respectively. The $\mathrm{FWHM}_{\mathrm{FEL}}$ is about $0.25 \mathrm{meV}$ and the spectral resolution of the Fourier-transform infrared spectrometer is $37 \mu \mathrm{eV}\left(0.3 \mathrm{~cm}^{-1}\right)$. The right upper graph shows the $\mathrm{Si}: \mathrm{Bi}$ absorption spectrum compared with the FEL pump spectrum.

lasing dynamics under almost resonant pumping into the $3 p_{0}$ Bi state at about $1 \mathrm{meV}$ off the resonance with the $2 p_{ \pm}$ $\mathrm{Bi}$ state. In this case PIL and SRS lasing have different frequencies and can be spectrally resolved (Fig. 6). At the start of the pump macropulse excited electrons relax from the $3 p_{0}$ state into the $2 p_{ \pm}$state and form population inversion. As a result, PIL on the $2 p_{ \pm} \rightarrow 1 s(E)$ transition 23.8) $\mathrm{meV}, 5.75 \mathrm{THz}$ ) sets on first. With about $1 \mu \mathrm{s}$ delay, SRS lasing appears at $24.5 \mathrm{meV}(5.92 \mathrm{THz})$. This can be clearly seen in the dynamics of the integrated intensity of the $\mathrm{Si}$ : Bi laser as a function of the FEL pump photon energy and by the $\mathrm{Si}: \mathrm{Bi}$ lasing emission spectra reconstructed from their time-resolved interferogram maps (see complementary files Si_Integrated_Emission.gif and Si_Emission_Spectrum.gif in the Supplemental Material [18]).

In conclusion, we have observed simultaneous lasing by two principally different and competing mechanisms operating in a degenerate four-level system in $\mathrm{Si}$ :Bi: conventional lasing, which requires population inversion between particular electronic levels, and inversion-less Raman lasing on the same levels. The interplay between SRS and conventional inversion-based lasing depends on the pump frequency and power. The high- $Q$ factor of the laser resonator enables the competition of both processes, because the photon lifetime in the resonator exceeds the ns intervals between two pump pulses. Under resonant pump conditions into the upper laser level the PIL mechanism starts earlier since its gain sustains over the approximately 50-ps lifetime of the laser upper level while the SRS gain acts only during the approximately 10-pslong pump pulse. Later on, the accumulation of the photons in the resonator leads to an increase of the SRS gain within the pump micropulse of the FEL and transition from population-inversion-based lasing to Raman lasing occurs so that for some time both lasing mechanisms exist simultaneously. This is caused by changes of the populations of the involved levels and thus the gain of both processes. At slight detuning of the pump wavelength from the resonance the lasing mechanisms can be distinguished by time-resolved spectroscopy. The observed lasing dynamics has been described by a rate equation model for a three-level system.

This work was partly supported by the GermanRussian "Research on technological advances of radiation sources of photons and neutrons based on accelerators and neutron sources in cooperation with research organizations and universities of the Federal Republic of Germany" (InTerFEL project, No. BMBF05K2014 and No. RFMEFl61614X0008), the Netherlandse Organisatie voor Wetenschappelijk Onderzoek (NWO), as well as joint German-Russian project supported by the Deutsche Forschungsgemeinschaft (Project No. 389056032) and the Russian Foundation for Basic Research (RFBF Project No. 18-502-12077-DFG). N. D. acknowledges support by the Helmholtz Research School on Security Technologies.

[1] M. O. Scully and M. Fleischhauer, Lasers Without Inversion, Science 263, 337 (1994).

[2] H. Rong, S. Xu, Y.-H. Kuo, V. Sih, O. Cohen, O. Raday, and M. Paniccia, Low-Threshold Continuous-Wave Raman Silicon Laser, Nat. Photonics 1, 232 (2007).

[3] M. Troccoli, A. Belyanin, F. Capasso, E. Cubukcu, D. L. Sivco, and A. Y. Cho, Raman Injection Laser, Nature (London) 433, 845 (2005).

[4] H. C. Liu, I. W. Cheung, A. J. SpringThorpe, C. Dharma-wardana, Z. R. Wasilewski, D. J. Lockwood, and G. C. Aers, Intersubband Raman Laser, Appl. Phys. Lett. 78, 3580 (2001).

[5] J. B. Khurgin, G. Sun, L. R. Friedman, and R. A. Soref, Comparative Analysis of Optically Pumped Intersubband Lasers and Intersubband Raman Oscillators, J. Appl. Phys. 78, 7398 (1995).

[6] J. B. Khurgin and H. C. Liu, Stimulated Polariton Scattering in Intersubband Lasers: Role of Motional Narrowing, Phys. Rev. B 74, 035317 (2006).

[7] A. Belyanin, M. Troccoli, and F. Capasso, in Intersubband Transitions in Quantum Structures, edited by R. Paiella (McGraw Hill, New York, 2006), Chap. 6, pp. 237-283. 
[8] M. Scheinert, H. Sigg, and S. Tsujino, Intersubband Raman Laser from GaInAs/AlInAs Double Quantum Wells, Appl. Phys. Lett. 91, 131108 (2007).

[9] O. Gauthier-Lafaye, F. H. Julien, S. Cabaret, and J.-M. Lourtioz, High-Power GaAs/AlGaAs Quantum Fountain Unipolar Laser Emitting at $14.5 \mu \mathrm{m}$ with $2.5 \%$ Tunability, Appl. Phys. Lett. 74, 1537 (1999).

[10] H. C. Liu, I. W. Cheung, A. J. SpringThorpe, C. Dharma-wardana, Z. R. Wasilewski, D. J. Lockwood, and G. C. Aers, Intersubband Raman Laser, Appl. Phys. Lett. 78, 3580 (2001).

[11] S. G. Pavlov, H.-W. Hübers, M. H. Rümmeli, R. Kh. Zhukavin, E. E. Orlova, V. N. Shastin, and H. Riemann, Far-Infrared Stimulated Emission from Optically Excited Bismuth Donors in Silicon, Appl. Phys. Lett. 80, 4717 (2002).

[12] S. G. Pavlov, U. Böttger, R. Eichholz, N. V. Abrosimov, H. Riemann, V. N. Shastin, B. Redlich, and H.-W. Hübers, Terahertz Lasing from Silicon by Infrared Raman Scattering on Bismuth Centers, Appl. Phys. Lett. 95, 201110 (2009).

[13] G. B. Wright and A. Mooradian, Raman Scattering from Donor and Acceptor Impurities in Silicon, Phys. Rev. Lett. 18, 608 (1967).
[14] N. Stavrias, K. Saeedi, B. Redlich, P. T. Greenland, H. Riemann, N. V. Abrosimov, M. L. W. Thewalt, C. R. Pidgeon, and B. N. Murdin, Competition between Homogeneous and Inhomogeneous Broadening of Orbital Transitions in Si:Bi, Phys. Rev. B 96, 155204 (2017).

[15] S. G. Pavlov, R. K. Zhukavin, V. N. Shastin, and H.-W. Hübers, The Physical Principles of Terahertz Silicon Lasers Based on Intracenter Transitions, Phys. Status Solidi (b) 250, 9 (2013).

[16] V. V. Tsyplenkov, E. V. Demidov, K. A. Kovalevsky, and V. N. Shastin, Relaxation of Excited Donor States in Silicon with Emission of Intervalley Phonons, Semiconductors 42 , 1016 (2008).

[17] S. G. Pavlov, H.-W. Hübers, J. N. Hovenier, T. O. Klaassen, D. A. Carder, P. J. Phillips, B. Redlich, H. Riemann, R. K. Zhukavin, and V. N. Shastin, Stimulated Terahertz Stokes Emission of Silicon Crystals Doped with Antimony Donors, Phys. Rev. Lett. 96, 037404 (2006).

[18] See Supplemental Material at http://link.aps.org/ supplemental/10.1103/PhysRevX.8.041003 for the theoretical calculations of dynamics of the lasing system as well as for the dynamic changes in the emission reconstructed from time-resolved measurements. 